\title{
THE FIRST SCIENCE RESULTS FROM SPHERE: DISPROVING THE PREDICTED BROWN DWARF AROUND V471 TAU*
}

\author{
A. Hardy ${ }^{1,6}$, M. R. Schreiber ${ }^{1,6}$, S. G. Parsons ${ }^{1}$, C. Caceres ${ }^{1,6}$, G. Retamales ${ }^{1,6}$, Z. Wahhaj ${ }^{2}$, D. Mawet $^{2}$, \\ H. Canovas ${ }^{1,6}$, L. Cieza ${ }^{5,6}$, T. R. Marsh ${ }^{3}$, M. C. P. Bours ${ }^{3}$, V. S. Dhillon ${ }^{4}$, and A. Bayo ${ }^{1,6}$ \\ ${ }^{1}$ Departamento de Física y Astronomía, Universidad Valparaíso, Avenida Gran Bretaña 1111, Valparaíso, Chile \\ ${ }^{2}$ European Southern Observatory, Casilla 19001, Vitacura, Santiago, Chile \\ ${ }^{3}$ Department of Physics, Gibbet Hill Road, University of Warwick, Coventry CV4 7AL, UK \\ ${ }_{5}^{4}$ Department of Physics and Astronomy, University of Sheffield, Sheffield S3 7RH, UK \\ ${ }^{5}$ Nucleo de Astronomia, Universidad Diego Portales, Av. Ejército 441, Santiago, Chile \\ ${ }^{6}$ Millennium Nucleus "Protoplanetary Disks in ALMA Early Science", Universidad de Valparaíso, \\ Avenida Gran Bretaña 1111, Valparaíso, Chile \\ Received 2014 December 17; accepted 2015 January 16; published 2015 February 18
}

\begin{abstract}
Variations of eclipse arrival times have recently been detected in several post-common envelope binaries consisting of a white dwarf and a main-sequence companion star. The generally favored explanation for these timing variations is the gravitational pull of one or more circumbinary substellar objects periodically moving the center of mass of the host binary. Using the new extreme-AO instrument SPHERE, we image the prototype eclipsing post-common envelope binary V471 Tau in search of the brown dwarf that is believed to be responsible for variations in its eclipse arrival times. We report that an unprecedented contrast of $\Delta m_{H}=12.1$ at a separation of 260 mas was achieved, but resulted in a non-detection. This implies that there is no brown dwarf present in the system unless it is 3 mag fainter than predicted by evolutionary track models, and provides damaging evidence against the circumbinary interpretation of eclipse timing variations. In the case of V471 Tau, a more consistent explanation is offered with the Applegate mechanism, in which these variations are prescribed to changes in the quadrupole moment within the main-sequence star.
\end{abstract}

Key words: binaries: close - binaries: eclipsing - brown dwarfs - planet-star interactions stars: individual (V471 Tau) - white dwarfs

\section{INTRODUCTION}

Circumbinary substellar objects, although anticipated for a long time, have only recently been identified around mainsequence binary stars (Doyle et al. 2011). Long before this, however, claims were made for circumbinary substellar objects around close white dwarf main-sequence binaries. Initially consisting of a main-sequence binary with separations of the order of 1 AU (Zorotovic \& Schreiber 2013), these systems are believed to have gone through a spectacular phase of binary star evolution to explain their current close separation. When the more massive star of the binary evolves off the main sequence, it fills its Roche-lobe and generates dynamically unstable mass transfer onto the secondary star. As the timescale for dynamically unstable mass transfer is much shorter than the thermal timescale of the secondary, the latter cannot adjust its structure fast enough to incorporate the overflowing mass. Instead, a common envelope of material forms around both the secondary star and the core of the giant. Drag forces between the envelope and the central binary then cause the envelope to be expelled at the expense of orbital energy and angular momentum of the binary (e.g., Webbink 1984; Zorotovic et al. 2010; Ivanova et al. 2013). The emerging white dwarf main-sequence binaries contain separations of just a few solar radii and are known as post-common envelope binaries (PCEBs; Nebot Gómez-Morán et al. 2011).

Shortly after the discovery of the first PCEB, it was realized that it displays variations in its eclipse arrival times. Today, similar variations are seen in almost all eclipsing PCEBs with

\footnotetext{
* Based on observations collected at the European Southern Observatory, Chile, program ID 60.A-9355(A).
}

sufficiently long coverage (Parsons et al. 2010b; Zorotovic \& Schreiber 2013), for which the most common hypothesis is the presence of a circumbinary object, typically a brown dwarf or multiple giant planets. In this scenario, the gravitational pull of the circumbinary objects periodically moves the center of mass of the host binary stars, thereby changing the light travel time of the eclipse signal to earth (Irwin 1959). Indeed, the planetary model employed to explain the eclipse timing variations (ETVs) seen in the PCEB NN Ser (Beuermann et al. 2010) successfully predicted new eclipse arrival times (Beuermann et al. 2013; Marsh et al. 2014), providing support to the circumbinary interpretation but raising questions regarding the formation of these third objects. Zorotovic \& Schreiber (2013) favor a scenario in which the circumbinary objects form as a consequence of the common envelope evolution, in a so-called second generation scenario. This is based on the finding that nearly all PCEBs with sufficiently long coverage show ETVs, yet only a small fraction of main-sequence binaries seem to host circumbinary substellar objects. Indeed, Schleicher \& Dreizler (2014) were able to develop a model in which a second generation protoplanetary disk forms during common envelope evolution and produces giant planets through the disk instability model. In contrast, Bear \& Soker (2014) prefer the first-generation scenario, in which the objects form at a similar time to their main-sequence hosts and survive the common-envelope phase. They claim that if a second-generation scenario were true, then too large a fraction of the common envelope mass would have to form into substellar companions.

However, before further investigating possible formation scenarios, we must exercise caution with the third-body 
hypothesis. Although the circumbinary object model has proved successful in the case of NN Ser, this is an exception. In general, the predictions from proposed planetary systems around PCEBs disagree with more recent eclipse timing measurements (Parsons et al. 2010b; Bours et al. 2014), and some of the proposed planetary systems are dynamically unstable on very short timescales (Horner et al. 2012, 2013; Wittenmyer et al. 2013). The failure of all circumbinary object models except that for NN Ser implies either that our timing coverage is insufficient, or that there must be an alternative mechanism driving ETVs.

To progress with this situation, it has become vital that the circumbinary companion interpretation be tested independently. The most conclusive way to achieve this is to image one of the proposed objects and the natural choice for such an observation is V471 Tau. V471 Tau consists of a $0.84 \pm 0.05$ $M_{\odot}$ white dwarf and a $0.93 \pm 0.07 M_{\odot}$ secondary star $\left(\mathrm{O}^{\prime}\right.$ Brien et al. 2001), and is a member of the 625 Myr old Hyades open cluster (Perryman et al. 1998). Soon after its discovery (Nelson \& Young 1970), Lohsen (1974) reported ETVs which have been interpreted as being caused by a circumbinary brown dwarf (Beavers et al. 1986; Guinan \& Ribas 2001). V471 Tau is ideal to test the circumbinary interpretation because it is nearby, bright, and the proposed brown dwarf reaches projected separations exceeding 200 mas, making detection possible with new extreme-AO facilities such as SPHERE (Beuzit et al. 2008).

Here we present new high-precision eclipse times of V471 Tau and use these to refine the proposed brown dwarf parameters using the Markov Chain Monte Carlo (MCMC) method. We then test the circumbinary interpretation of ETVs with SPHERE science verification observations, with sufficiently high contrast to detect the brown dwarf independent of whether it formed in a second- or first-generation scenario.

\section{OBSERVATIONS AND DATA REDUCTION}

\subsection{High-speed Eclipse Photometry}

In order to refine the orbit of the circumbinary companion, we obtained high-speed photometry of the eclipse of the white dwarf in V471 Tau with the frame-transfer camera ULTRACAM (Dhillon et al. 2007) mounted as a visitor instrument on the $3.6 \mathrm{~m}$ New Technology Telescope on La Silla in 2010 November and December. ULTRACAM uses a triple beam setup allowing one to obtain data in the $u^{\prime}, g^{\prime}$, and $i^{\prime}$ bands simultaneously with negligible dead time between frames. Due to the brightness of the target, we de-focussed the telescope and used exposure times of $\sim 5 \mathrm{~s}$. There were no suitably bright nearby stars in ULTRACAM's field of view to use for comparison. We therefore used the $i^{\prime}$-band observations where the eclipse is extremely shallow as a comparison source for the $u^{\prime}$-band data. This results in a large color term in the resulting light curve, but does remove variations in the conditions and does not affect the eclipse timings. In some observations, the conditions were good enough that no comparison source was required.

These data were reduced using the ULTRACAM pipeline software (Dhillon et al. 2007) and the resultant eclipse light curves were fitted with a dedicated code designed to fit binaries containing white dwarfs (Copperwheat et al. 2010). The
Table 1

ULTRACAM Eclipse Times for V471 Tau

\begin{tabular}{llc}
\hline \hline Cycle & HMJD(TDB) & Uncertainty (seconds) \\
\hline 2848 & 5512.2840584 & 1.76 \\
2886 & 5532.0889885 & 1.59 \\
2911 & 5545.1185942 & 1.62 \\
2915 & 5547.2033608 & 2.37 \\
\hline
\end{tabular}

measured eclipse times were then heliocentrically corrected and are listed in Table 1.

\subsection{SPHERE Observations}

The imaging data testing the existence of the brown dwarf were acquired using the extreme adaptive optics instrument SPHERE at the UT3 Nasmyth focus of the VLT, on 2014 December 11. An earlier set of observations was performed on 2014 December 6, but is not used here because of poorer data quality. SPHERE is able to provide $\mathrm{H}$ - and K-band images with Strehl ratios $>90 \%$. The integral field spectrograph (IFS) and infrared dual-band imager and spectrograph (IRDIS) were used simultaneously in the IRDIFS mode. The IFS delivered a datacube containing 38 monochromatic images at a spectral resolution of $\mathrm{R} \sim 50$ between 0.96 and $1.34 \mu \mathrm{m}$, while IRDIS delivered dual-band imaging in the $\mathrm{H} 2$ and $\mathrm{H} 3$ bands (central wavelengths of 1.59 and $1.67 \mu \mathrm{m}$, respectively, and FWHMs of 0.0531 and $0.0556 \mu \mathrm{m})$.

The brightness of the target and the desired contrast required the use of the $\mathrm{N}_{-}$ALC_YJH coronagraph with an inner working angle of $0 \overline{\prime \prime} 15$. Detector integration times were set at $64 \mathrm{~s}$ for each detector. The observations were obtained in pupilstabilized mode where the field rotates. To derotate and combine the images, one needs to accurately measure the center of rotation which is also the location of the star behind the coronagraph. To achieve this, a waffle pattern was introduced into the deformable mirror of the AO system, placing four replicas of the star equidistant from it in a square pattern. These calibration images were taken before and after the science, allowing subpixel accuracy in centroiding. Offcoronagraph, unsaturated images of the star were also obtained with a neutral density filter to allow sensitivity/contrast measurements. Basic reduction, including dark and flat-fielding was performed using the SPHERE pipeline. Custom angular differential imaging (ADI) routines, particularly for subpixel shifting and aligning of speckles, were used to subtract the star (Wahhaj et al. 2013). A custom principal component analysis routine was also applied to the data to compare with the ADI reduction (Mawet et al. 2013).

\section{PREDICTING THE PROJECTED SEPARATION OF THE POTENTIAL BROWN DWARF}

Assuming a third body orbiting around V471 Tau, the time delay or advance caused by this body can be expressed as

$$
\Delta T=\frac{a_{12} \sin i_{3}}{c}\left[\frac{1-e_{3}^{2}}{1+e_{3} \cos \nu_{3}} \sin \left(\nu_{3}+\omega\right)+e_{3} \sin (\omega)\right](1)
$$

(e.g., Irwin 1959), where $c$ is the speed of light and $a_{12}$ is the semimajor axis of the binary star's orbit around the common center of mass of the triple system. The other parameters define the orbit of the third body, i.e., its inclination $i_{3}$, the orbital 


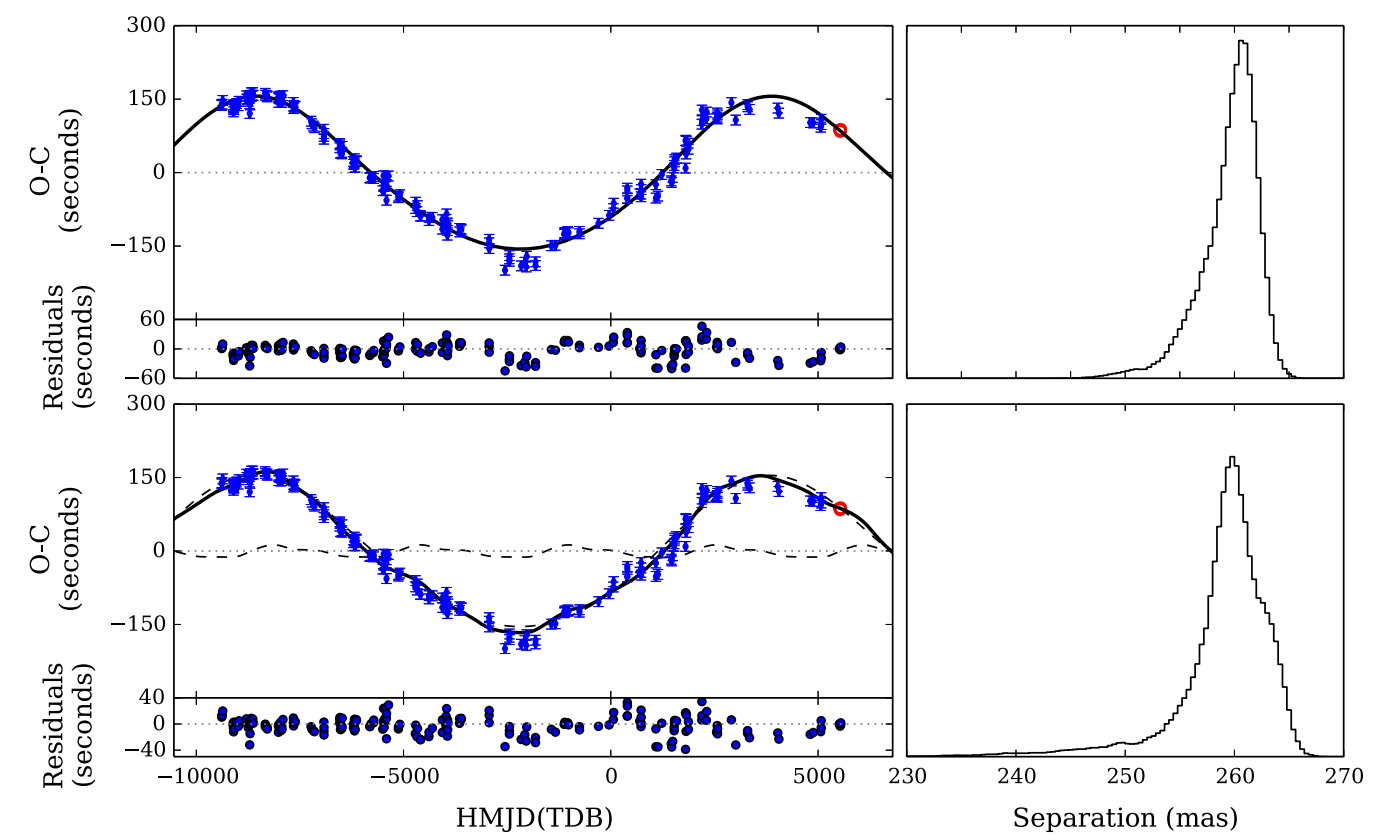

Figure 1. Observed-minus-calculated $(O-C)$ eclipse arrival times for V471 Tau. Upper left panel: the eclipse times and associated errors with the high-precision eclipse times reported in this paper shown as the red open circles. The best-fit model assuming a third-body perturbation is shown as the solid black line. The residuals of this fit can be seen directly beneath the curve. Upper right panel: the projected separations predicted by the MCMC simulation for observation in 2014 December, assuming a distance of $50 \mathrm{pc}$ to V471 Tau. The lower panels are identical, but denote the results of a two body fit to the the eclipse timing variations. The dashed lines in the lower left panel denote the contributions from the different objects with their sum shown in black. Despite the extra body, the predicted separation and mass of the brown dwarf hardly change.

eccentricity $e_{3}$, the argument of periastron $\omega$, and the true anomaly $\nu_{3}$.

As shown by Marsh et al. (2014), strong correlations can exist between orbital parameters and the problem is highly degenerate unless a large number of high-precision eclipse timing measurements are available. Only our recent ULTRACAM measurements provide precise eclipse timings, with uncertainties of $\sim 1.8 \mathrm{~s}$, whereas the timings in the literature have been assigned large error estimates of $15 \mathrm{~s}$ for the sake of caution. To properly identify not only the best-fit parameters but also their uncertainties, we performed an MCMC simulation to the eclipse times.

The prediction of the best-fit model can be seen in Figure 1 (top left panel) alongside all of the archival observed-minuscalculated eclipse times (Kundra \& Hric 2011) and the new times reported in this paper. This best-fit model corresponds to a brown dwarf of mass $0.044 \pm 0.001 M_{\odot}$ and semimajor axis $12.8 \pm 0.16 \mathrm{AU}$.

While the parameters of the brown dwarf in the one body fit are well constrained by the eclipse times (Figure 2), the residuals are far from random and suggest that another mechanism may also be at work. To test this possibility, we performed another MCMC with two companions to account for these deviations. This further allowed us to test whether the brown dwarf causing the main variation could be at a smaller separation or be less massive, which would make it harder to detect. The resulting best fit is shown in the bottom left panel of Figure 1.

The derived orbital parameters for both cases were then projected onto the sky using a distance to V471 Tau of $\sim 50 \mathrm{pc}$, as measured by Hipparcos (van Leeuwen 2007), to obtain a predicted separation for the brown dwarf companion in 2014 December. The simulation suggests a separation of $260_{-19}^{+6}$ mas for the one body fit and this value hardly changes if an additional object is assumed to account for the problems of the one body fit (see Figure 1, right panels).

\section{TESTING THE PREDICTION WITH SPHERE}

No third component is present in the SPHERE IRDIS images (Figure 3, left panel). The contrast achieved was estimated via two different methods of injection of fake companions. In the first, fake companions of a known contrast were injected at different angular separations, and the contrast defined by where the fake companion was recovered $95 \%$ of the time (Wahhaj et al. 2013). In the second method, the fake companions were used to measure the post-ADI throughput loss, and this was used to renormalize the contrast curve of a typical saturated SPHERE PSF. In both cases, the $\mathrm{H} 2$ and $\mathrm{H} 3$ channels were summed as no spectral difference between the channels was expected, and the curves were corrected for small-sample statistics at small separations (Mawet et al. 2014). The resulting contrast curves for the IRDIS detector can be seen in Figure 3, right panel, with the former method shown as the solid line and the latter as the dashed. There is good agreement between the two methods at the predicted separation of $\sim 260$ mas, and both indicate an achieved contrast of $\sim 12.1 \mathrm{mag}$. To determine if this is sufficient indeed to detect the brown dwarf, it is necessary to know its age, mass, and metallicity, from which the brown dwarf luminosity can be predicted. We find the mass is well constrained from the MCMC models, and the metallicity was assumed identical to other members of the Hyades cluster with $[M / H]=0.14 \pm$ 0.05 (Perryman et al. 1998).

The cooling age of the white dwarf in V471 Tau is $\sim 10$ Myrs, and places a stringent constraint on the age in a 

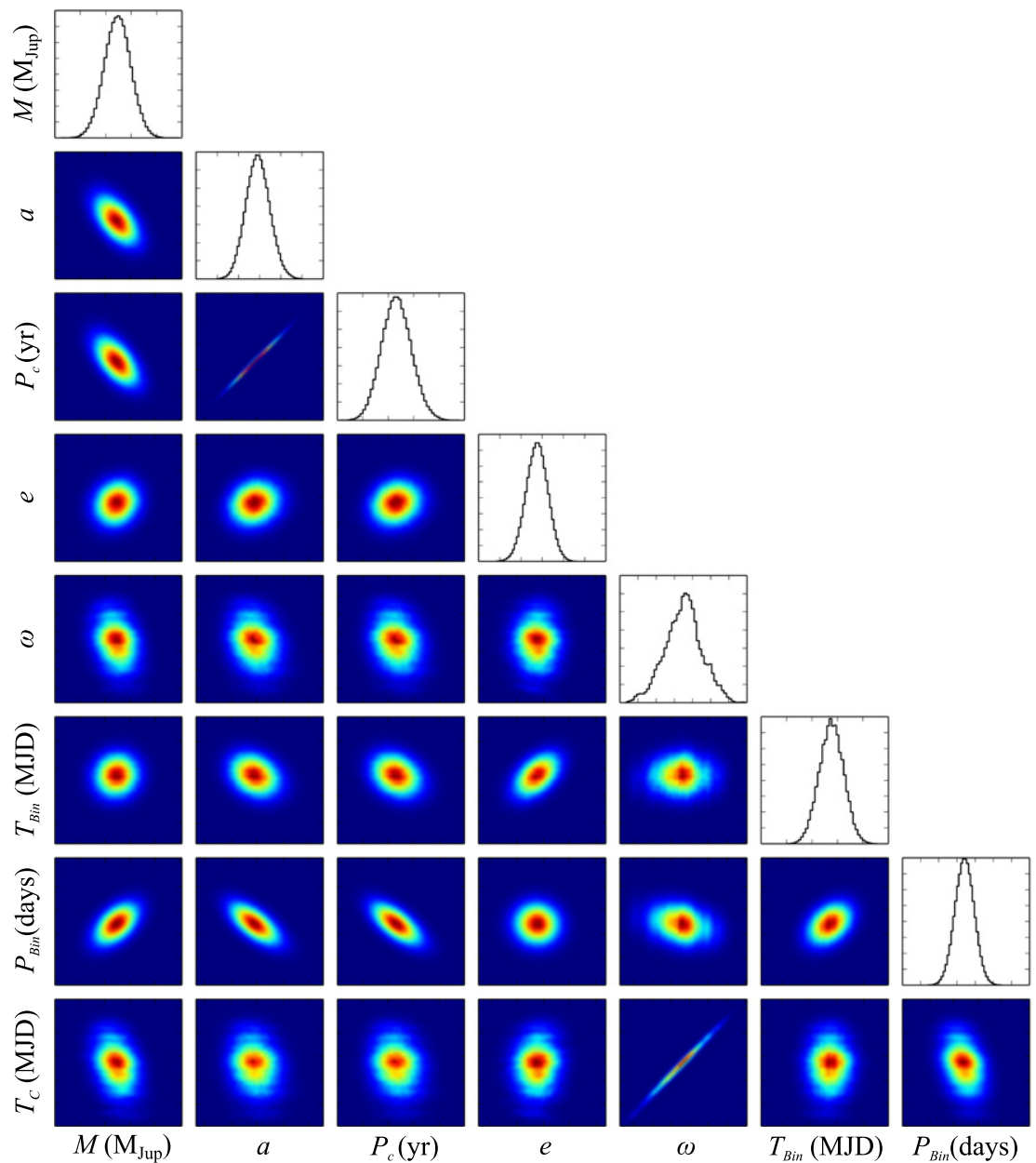

$T_{B i n}(\mathrm{MJD})$

$P_{B i n}($ days $)$

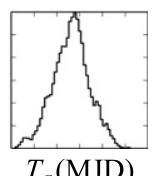

Figure 2. Correlations and histograms for the MCMC simulation. $M$ is the mass of the brown dwarf, $a$ the semimajor axis of its orbit, $P_{C}$ the period, $e$ the eccentricity, $\omega$ the argument of periastron, $T_{B i n}$ the time of periastron of the binary, $P_{B i n}$ the period of the binary, and $T_{C}$ the time of periastron of the brown dwarf. All parameters appear well constrained with no significant correlations.
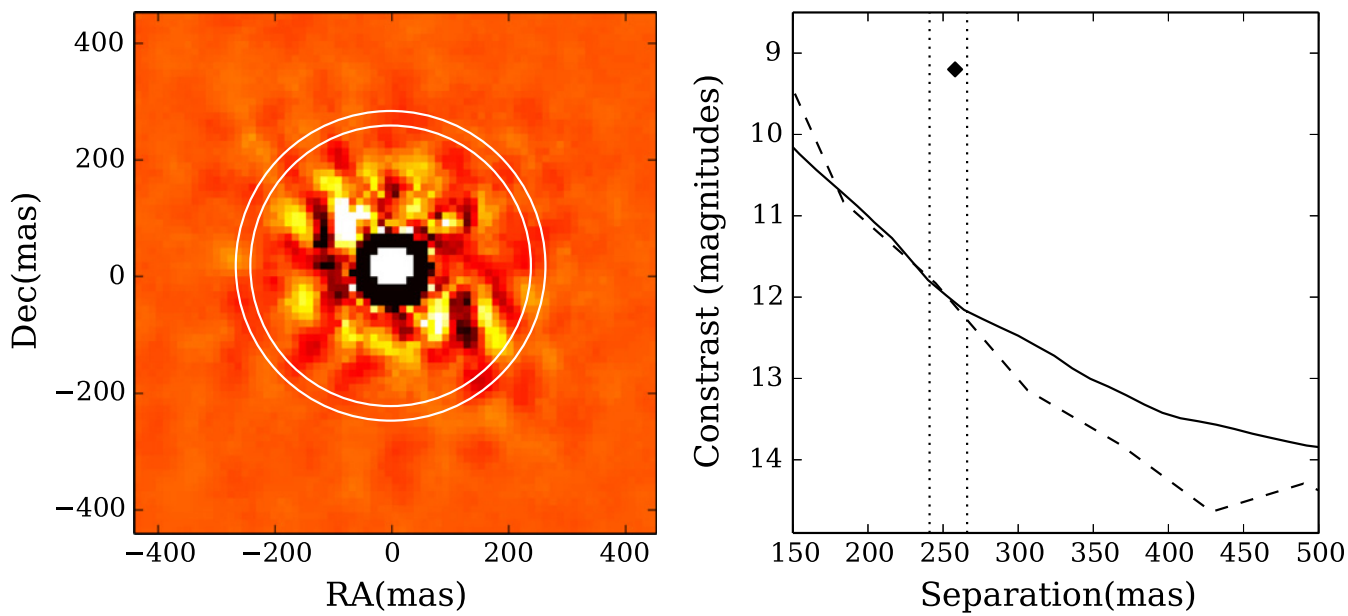

Figure 3. H-band image of V471 Tau obtained on the SPHERE IRDIS instrument at VLT. Left panel: resulting image after angular differential imaging (ADI). The area in-between the white circles denotes the $5 \sigma$ predicted position of the brown dwarf. Right panel: contrast curves obtained via two different methods of fake companion injection (see the text for details). The vertical lines again denote the predicted position of the object with the diamond denoting the predicted contrast of a first generation brown dwarf.

second generation scenario. If the $0.044 M_{\odot}$ brown dwarf had formed in such a scenario, BTSettl models (Allard et al. 2012) combined with isocrones from Baraffe et al. (1998) predict a $\Delta m_{H} \sim 4.5$. This is 7.5 mag brighter than our detection limit, conclusively ruling out a second generation formation scenario for the potential brown dwarf around V471 Tau.

If the brown dwarf formed in a first generation scenario, we can obtain an estimate of its age from the age of the Hyades 
cluster (625 Myr). An identical modeling procedure suggests that such a brown dwarf will have a contrast of $\Delta m_{H} \sim 9.2$ in the $\mathrm{H}$ band, 3 mag brighter than our detection limit.

\section{DISCUSSION}

\subsection{Could the BD Have Escaped Our SPHERE Observations?}

Inspecting the contrast curve presented in Figure 3, it is clear that if a first generation brown dwarf was at a smaller separation and/or had a lower mass, it may still have escaped detection. To test whether a brown dwarf could escape detection while simultaneously causing the $O-C$ variations, we performed a final MCMC simulation with separations limited to only those which would be undetectable by SPHERE. The resulting fit drastically failed to explain the $O-C$ data, and delivered a $\chi_{\text {red }}^{2}$ of 47.7 , compared to 1.1 in the case of the constraint-free brown dwarf. As shown in Figure 1 (lower left panel), not even the addition of an extra body can cause the brown dwarf to reach a separation undetectable by SPHERE. In order to recreate the observed $O-C$ variations, a large mass, high separation companion seems to be needed, and we therefore find no configuration in which the brown dwarf would have escaped SPHERE.

The second possibility as to how the brown dwarf might have escaped detection is that the models of substellar evolutionary tracks are incorrect. Indeed, if the $0.044 M_{\odot}$ brown dwarf was at a temperature of $\sim 700 \mathrm{~K}$, as opposed to the $\sim 1500 \mathrm{~K}$ predicted for a first-generation brown dwarf, it would escape detection. However, despite the uncertainties associated with these cooling models, there is no evidence to suggest models are off to this degree (Bell et al. 2012; Lodieu et al. 2014).

Finally, the brown dwarf around V471 Tau might be unique because of the evolutionary history of the host binary star. One might, for example, speculate that it accreted significant amounts of material during the rather recent common envelope phase, and this could have caused its metallicity to deviate significantly from the metallicity of the Hyades. Indeed, if the brown dwarf were first generation and possessed a metallicity $[M / H]=0.5$, then models predict it would not be detected in the presented observations (Allard et al. 2012). However, the metallicity of the secondary K star has been studied, and found to show no peculiar abundances despite the possibility it accreted material during the common envelope phase (Still \& Hussain 2003). It is therefore highly unlikely that the brown dwarf metallicity is high enough to explain the non-detection. A final effect of recent accretion could be that the infalling mass made the brown dwarf fainter due to contraction (Baraffe et al. 2009), although confirming if this is indeed possible will require detailed evolutionary brown dwarf models dedicated to V471 Tau, which is beyond the scope of the present paper.

\subsection{Applegate's Mechanism}

If our non-detection is due to the lack of a brown dwarf, then an alternative mechanism must be responsible for the ETVs. Currently, there are two such alternative theories for ETVs in PCEBS. The first, apsidal precession, can confidently be ruled out for V471 Tau. This mechanism prescribes ETVs to the time dependance in the argument of periastron. Although apsidal precession would require an eccentricity in the binary of just $e=0.01$ to create the $\sim 300$ s timing variations seen in Figure 1, the timescale would be less than $3 \mathrm{yr}$, and not $\sim 30 \mathrm{yr}$ as observed. The second alternative explanation, the Applegate mechanism (Applegate 1992), is potentially able to drive the variations of the eclipse arrival times seen in V471 Tau. This theory prescribes these variations to quasi-periodic oblateness changes in the main-sequence star, presumed to be driven by solar-like magnetic cycles. The $\mathrm{K}$ star in V471 Tau is particularly active, and may provide sufficient energy to drive these variations. Applegate's mechanism is therefore currently the most convincing explanation for the eclipse arrival times observed in V471 Tau.

However, although it is a suitable explanation in the case of V471 Tau, in almost all other PCEBs showing ETVs, a classical Applegate's mechanism can be ruled out as their main-sequence stars tend to be less active. NN Ser is one such system, and currently only the proposed planetary system can explain its behavior (Brinkworth et al. 2006; Parsons et al. 2010a; Parsons et al. 2014), although it is possible that an asyet unconsidered model of magnetic field variations could still offer an explanation. Thus, Applegate does not offer a comprehensive explanation for ETVs, and confirmation of its effect in V471 Tau is needed alongside additional tests of the third-body interpretation in other systems.

\section{CONCLUSION}

We have presented deep SPHERE science verification observations of V471 Tau testing the hypothesis that the observed ETVs are caused by a circumbinary brown dwarf. We reached an excellent contrast of $\Delta m_{H}=12.1$ at the predicted separation of the brown dwarf but no companion can be seen in the images. This excludes both a brown dwarf formed in a second generation scenario, as well as a standard brown dwarf at the age of the Hyades cluster to be present around V471 Tau. The Applegate mechanism is hence the one and only remaining model currently explaining the ETVs seen in V471 Tau.

With this result, the origin of ETVs in PCEBs remains a puzzle. While no theory but the existence of two circumbinary planets can currently explain the variations seen in the PCEB $\mathrm{NN}$ Ser, the most reasonable explanation for the variations seen in V471 Tau is now the Applegate mechanism. We therefore conclude that in their current form, neither the third body interpetation nor Applegate's mechanism offer a general explanation for the ETVs observed in nearly all PCEBs.

A.H., M.R.S., C.C., H.C., L.C., and A.B. acknowledge support from the Millennium Nucleus RC130007 (Chilean Ministry of Economy). M.R.S., S.P., C.C., L.C., and A.B. also acknowledge support from FONDECYT grants 1141269, $3140585,3140592,1440109$, and 11140572, respectively. T.R.M. thanks the UK's Science and Technology Facilities Council for support during the course of this work under grant ST/L000733/1.

Facilities: VLT: Melipal (SPHERE), NTT (ULTRACAM).

\section{REFERENCES}

Allard, F., Homeier, D., \& Freytag, B. 2012, RSPTA, 370, 2765

Applegate, J. H. 1992, ApJ, 385, 621

Baraffe, I., Chabrier, G., Allard, F., \& Hauschildt, P. H. 1998, A\&A, 337, 403

Baraffe, I., Chabrier, G., \& Gallardo, J. 2009, ApJL, 702, L27

Bear, E., \& Soker, N. 2014, MNRAS, 444, 1698

Beavers, W. I., Lui, A., \& Herczeg, T. J. 1986, ApJ, 300, 785

Bell, C. P. M., Naylor, T., Mayne, N. J., Jeffries, R. D., \& Littlefair, S. P. 2012, MNRAS, 424, 3178

Beuermann, K., Dreizler, S., \& Hessman, F. V. 2013, A\&A, 555, A133 
Beuermann, K., Hessman, F. V., Dreizler, S., et al. 2010, A\&A, 521, L60 Beuzit, J.-L., Feldt, M., Dohlen, K., et al. 2008, Proc. SPIE, 7014, 701418 Bours, M. C. P., Marsh, T. R., Breedt, E., et al. 2014, MNRAS, 445, 1924 Brinkworth, C. S., Marsh, T. R., Dhillon, V. S., \& Knigge, C. 2006, MNRAS, 365,287

Copperwheat, C. M., Marsh, T. R., Dhillon, V. S., et al. 2010, MNRAS, 402,1824

Dhillon, V. S., Marsh, T. R., Stevenson, M. J., et al. 2007, MNRAS, 378, 825

Doyle, L. R., Carter, J. A., Fabrycky, D. C., et al. 2011, Sci, 333, 1602

Guinan, E. F., \& Ribas, I. 2001, ApJL, 546, L43

Horner, J., Hinse, T. C., Wittenmyer, R. A., Marshall, J. P., \& Tinney, C. G. 2012, MNRAS, 427, 2812

Horner, J., Wittenmyer, R. A., Hinse, T. C., et al. 2013, MNRAS, 435, 2033

Irwin, J. B. 1959, AJ, 64, 149

Ivanova, N., Justham, S., Chen, X., et al. 2013, A\&A, 21, 59

Kundra, E., \& Hric, L. 2011, Ap\&SS, 331, 121

Lodieu, N., Boudreault, S., \& Béjar, V. J. S. 2014, MNRAS, 445, 3908

Lohsen, E. 1974, A\&A, 36, 459

Marsh, T. R., Parsons, S. G., Bours, M. C. P., et al. 2014, MNRAS, 437, 475

Mawet, D., Absil, O., Delacroix, C., et al. 2013, A\&A, 552, L13
Mawet, D., Milli, J., Wahhaj, Z., et al. 2014, ApJ, 792, 97

Nebot Gómez-Morán, A., Gänsicke, B. T., Schreiber, M. R., et al. 2011, A\&A, 536, A43

Nelson, B., \& Young, A. 1970, PASP, 82, 699

O’Brien, M. S., Bond, H. E., \& Sion, E. M. 2001, ApJ, 563, 971

Parsons, S. G., Marsh, T. R., Copperwheat, C. M., et al. 2010a, MNRAS, 402, 2591

Parsons, S. G., Marsh, T. R., Copperwheat, C. M., et al. 2010b, MNRAS, 407, 2362

Parsons, S. G., Marsh, T. R., Bours, M. C. P., et al. 2014, MNRAS, 438, L91

Perryman, M. A. C., Brown, A. G. A., Lebreton, Y., et al. 1998, A\&A, 331, 81

Schleicher, D. R. G., \& Dreizler, S. 2014, A\&A, 563, A61

Still, M., \& Hussain, G. 2003, ApJ, 597, 1059

van Leeuwen, F. 2007, A\&A, 474, 653

Wahhaj, Z., Liu, M. C., Biller, B. A., et al. 2013, ApJ, 779, 80

Webbink, R. F. 1984, ApJ, 277, 355

Wittenmyer, R. A., Horner, J., \& Marshall, J. P. 2013, MNRAS, 431, 2150

Zorotovic, M., \& Schreiber, M. R. 2013, A\&A, 549, A95

Zorotovic, M., Schreiber, M. R., Gänsicke, B. T., \& Nebot Gómez-Morán, A. 2010, A\&A, 520, A86 